\title{
Reliability and Generalizability of Similarity-Based Fusion of MEG and fMRI Data in Human Ventral and Dorsal Visual Streams
}

\author{
Yalda Mohsenzadeh ${ }^{1, *,+} \oplus$, Caitlin Mullin ${ }^{1,+}$, Benjamin Lahner ${ }^{1,2}$, Radoslaw Martin Cichy ${ }^{3}$ \\ and Aude Oliva ${ }^{1}$ \\ 1 Computer Science and AI Lab., Massachusetts Institute of Technology, Cambridge, MA 02139, USA; \\ crmullin@csail.mit.edu (C.M.); oliva@mit.edu (A.O.) \\ 2 Department of Biomedical Engineering, Boston University, Boston, MA 02215, USA; blahner@bu.edu \\ 3 Department of Education and Psychology, Freie Universität Berlin, Berlin 14195, Germany; \\ rmcichy@zedat.fu-berlin.de \\ * Correspondence: yalda@mit.edu \\ + These authors contributed equally.
}

Received: 22 October 2018; Accepted: 13 January 2019; Published: 10 February 2019

\begin{abstract}
To build a representation of what we see, the human brain recruits regions throughout the visual cortex in cascading sequence. Recently, an approach was proposed to evaluate the dynamics of visual perception in high spatiotemporal resolution at the scale of the whole brain. This method combined functional magnetic resonance imaging (fMRI) data with magnetoencephalography (MEG) data using representational similarity analysis and revealed a hierarchical progression from primary visual cortex through the dorsal and ventral streams. To assess the replicability of this method, we here present the results of a visual recognition neuro-imaging fusion experiment and compare them within and across experimental settings. We evaluated the reliability of this method by assessing the consistency of the results under similar test conditions, showing high agreement within participants. We then generalized these results to a separate group of individuals and visual input by comparing them to the fMRI-MEG fusion data of Cichy et al (2016), revealing a highly similar temporal progression recruiting both the dorsal and ventral streams. Together these results are a testament to the reproducibility of the fMRI-MEG fusion approach and allows for the interpretation of these spatiotemporal dynamic in a broader context.
\end{abstract}

Keywords: spatiotemporal neural dynamics; vision; dorsal and ventral streams; multivariate pattern analysis; representational similarity analysis; fMRI; MEG

\section{Introduction}

To solve visual object recognition, the human brain has developed a particular cortical topology within the ventral and dorsal streams, recruiting regions in cascading sequence, to quickly build a representation of what we see (i.e., [1-9]).

In order to reveal the complex neural dynamics underlying visual object recognition, neural representations must be resolved in both space and time simultaneously [10-15]. Towards this aim, Cichy and collaborators proposed a novel approach to combine functional magnetic resonance imaging (fMRI) with magnetoencephalography (MEG) termed MEG-fMRI fusion [8,9,16-18]. The results revealed the dynamics of the visual processing cascade. Neural representations first emerge in the occipital pole (V1, V2, V3) at around $80 \mathrm{~ms}$, and then progress in the anterior direction along the ventral (i.e., lateral-occipital cortex LO, ventral occipital cortex $\mathrm{VO}$, temporal occipital cortex $\mathrm{TO}$ 
and parahippocampal cortex PHC) and dorsal (intraparietal sulcus regions) visual streams within 110-170 ms after image onset.

The consistency of these results with established findings [19-26] suggests that fMRI-MEG fusion is an appropriate analytical tool to non-invasively evaluate the spatiotemporal mechanisms of perception. To assess the power of this technique to yield consistent results on visual recognition dynamics in the human ventral and dorsal streams [27-29], here we replicate the standard design and analysis protocol of fMRI-MEG fusion [9]. Specifically, we first evaluated the reliability of the fusion method at capturing the spatiotemporal dynamics of visual perception by assessing the neural agreement of visually similar experiences within individuals, asking: 'Do similar visual experiences obey similar spatiotemporal stages in the brain?' Given the known variation across brain regions for separate visual category input (e.g., [1,30-35]), our second objective was to determine the generalizability of these patterns: 'For a given task, which spatiotemporal properties are reproducible across diverse visual input and independent observer groups?'

The current results reveal that the established fusion method is reliable and generalizable within and across image sets and participant groups. In addition, novel approaches to region-of-interest based analyses validate the replicability of the spatio-temporal dynamics for highly similar visual content, demonstrating the robustness of the technique for tracing representational similarity in the brain over space and time.

\section{Materials and Methods}

This paper presents two independent experiments in which fMRI and MEG data were acquired when observers look at pictures of natural images. The fMRI and MEG data of Experiment 1 are original to this current work. Data of Experiment 2 has been published originally in [9] (Experiment 2).

\subsection{Participants}

Two separate groups of fifteen right-handed volunteers with normal or corrected to normal vision participated in Experiment 1 ( 9 female, $27.87 \pm 5.17$ years old) and Experiment 2 ( 5 female, $26.6 \pm 5.18$ years old, see [9]). The participants signed an informed consent form and were compensated for their participation. Both studies were conducted in accordance with the Declaration of Helsinki and approved by the Institutional Review Board of Massachusetts Institute of Technology (Approval code: 1510287948).

\subsection{Stimulus Set}

In Experiment 1, the stimulus set consisted of twin sets of 78 real-world natural images each (156 images total) from the LaMem dataset [36]. Twin-set 1 and Twin-set 2 each contained an image identifiable by the same verbal semantic description of the main object shown (based on consensus among the authors; see Figure $1 \mathrm{a}$ for examples). The sets were not significantly different on a collection of low level image statistics [37-39] (See Appendix A for more information about stimulus set). The stimulus set of Experiment 2 consisted of 118 natural images of objects [9] from the ImageNet dataset [40]. In both experiments, participants performed the same orthogonal vigilance task. See Appendix A for details on the Experimental Design.

\section{3. fMRI and MEG Acquisition}

MEG and fMRI data for Experiment 1 were acquired in separate sessions, similar to Experiment 2 in [9]. Images were presented $500 \mathrm{~ms}$ in all conditions. See Appendix B for data acquisition detail of both experiments.

\subsection{Data Analyses}

We performed several data analyses to test the robustness and generalizability of our results. First, we performed a full brain fMRI-MEG fusion $[8,9,16,17]$, which uses representational similarity 
analysis [41,42] to map MEG and fMRI data into a common space (see Appendix C for details). To summarize, the MEG data were analyzed in a time-resolved manner with $1 \mathrm{~ms}$ resolution. MEG sensor data at each time point were arranged in pattern vectors for each stimulus condition and repetition. To overcome computational complexity and reduce noise, trials per image condition were randomly sub-averaged in groups of 3 in Experiment 1, and groups of 5 in Experiment 2. These vectors were then used to train support vector machines to classify each pair of conditions. The performance of the binary SVM classifiers computed with leave-one-out cross validation procedure were interpreted as a pairwise dissimilarity measure (higher decoding indicates larger dissimilarity) and used to construct a condition by condition representational dissimilarity matrix (RDM) per time point (Figure 1b). The fMRI data were analyzed with a searchlight approach to construct the representational dissimilarity matrices in a voxel-resolved fashion. At every voxel in the brain, the condition-specific voxel patterns in its vicinity were extracted and pairwise condition-specific dissimilarities (1-Pearson's R) were computed to create a condition by condition fMRI RDM assigned to that voxel (Figure 1c). Then, in the similarity space of RDMs, MEG and fMRI data were directly compared (Spearman's R) to integrate high spatial resolution of fMRI with high temporal resolution of MEG. In detail, for a given time point the MEG RDM was correlated with fMRI RDMs specified with the searchlight method resulting in a 3D correlation map. Repeating the procedure for all time points as depicted in Figure 1d resulted in a $4 \mathrm{D}$ spatiotemporal correlation map per individual. The correlation maps were averaged over individuals and significant correlations were determined with permutation tests and multiple comparison corrections over time and space with cluster correction method $(\mathrm{N}=15$ in each experiment; cluster-definition threshold of 0.001 and cluster size threshold of 0.01 ). The detail on statistical tests are presented in Appendix F.

a
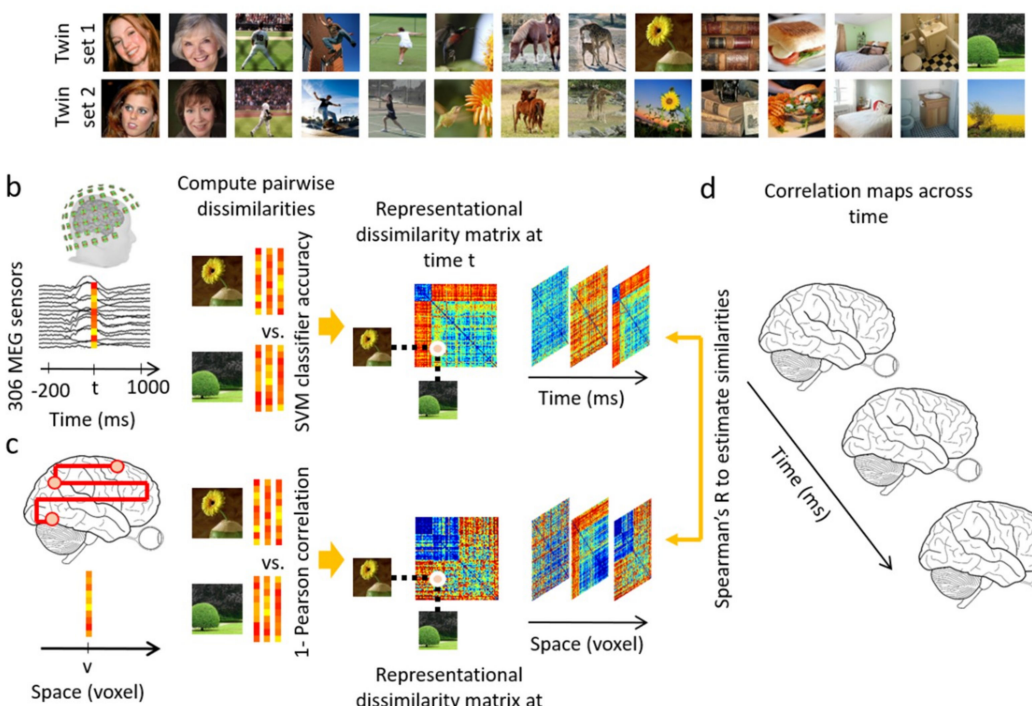
Compute pairwise dissimilarities

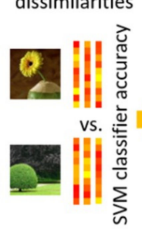

Representational

d Correlation maps across dissimilarity matrix at time
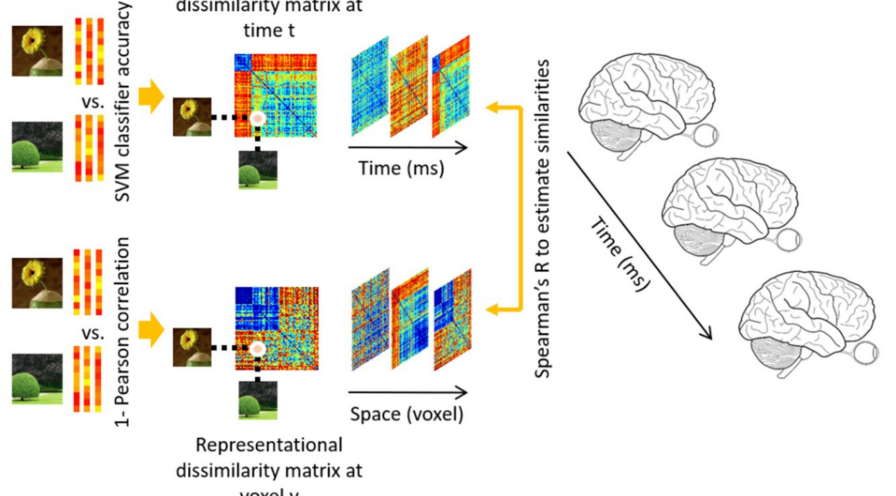

Representational
dissimilarity matrix

voxel v

Figure 1. Examples of stimuli of Experiment 1 and analysis scheme. (a) Examples of experimental condition: The stimulus set consisted of two sets (termed twin-sets 1 and 2) of 78 object images each ordered in pairs, such that each set was of the same semantic content but different in appearance. (b) MEG multivariate analysis. MEG data were extracted from $-200 \mathrm{~ms}$ to $1000 \mathrm{~ms}$ with respect to stimulus onset. The 306-dimensional MEG sensor data at each time point $t$ were arranged in vectors. For each pair of conditions, the performance of an SVM classifier in discriminating the conditions based on vector patterns was used as a measure of dissimilarity between the pair of conditions. A representational dissimilarity matrix at each time point $t$ was populated using these pairwise dissimilarities; (c) fMRI multivariate analysis. At each voxel $v$, the activation patterns in a sphere with radius of 4 voxels were extracted and pairwise dissimilarities of conditions were computed (1-Pearson's $\mathrm{R})$ yielding a representational dissimilarity matrix assigned to the voxel $\mathrm{v}$ at the center of the sphere; 
(d) fMRI-MEG similarity-based fusion. The MEG RDM representation at each time point $t$ were compared with the fMRI RDM representations at each voxel v by computing Spearman's R correlations. These computations resulted in correlation maps over the whole brain and across time.

Second, to quantitatively compare the spatiotemporal neural dynamics within and across the experiments and show how reliable they are, we performed two types of anatomically-defined region-of-interest (ROI) based analysis along ventral and dorsal streams (see Appendix D for detail): (1) a spatially restricted searchlight voxel-wise analysis: in this analysis, the searchlight based MEG-fMRI fusion correlation time series are averaged over voxels within an ROI; (2) the conventional ROI-based analysis: in this analysis, the condition-specific voxel responses within an ROI are extracted, and the pairwise condition-specific response dissimilarities are computed to create an ROI RDM which is then compared with the time-resolved MEG RDMs resulting in correlation time series (as in [9]).

\section{Results}

\subsection{Reliability of Similarity-Based fMRI-MEG Fusion Method}

We first assessed the reliability of the fMRI-MEG similarity-based data fusion method. For this we applied fMRI-MEG fusion separately to the two sets of images making up the Twins-set (i.e., Twin-set 1 and Twin-set 2) and compared the results.

Figures 2 and 3 show the spatiotemporal dynamics of visual perception in the ventral and dorsal visual pathways respectively for the Twins-sets over the time course of $1000 \mathrm{~ms}$. Qualitatively examining these results reveal that the neural representations start at the occipital pole around 70-90 ms after stimulus onset, followed by neural representations in the anterior direction along the ventral stream (Figure 2a,c), and along the dorsal stream up to the inferior parietal cortex (Figure 3a,c).

To quantitatively compare the spatiotemporal fusion maps between the Twin-sets we performed spatially restricted voxel-wise fMRI-MEG fusion on five regions-of-interest (ROIs) (early visual cortex (EVC), ventral occipital area (VO), parahippocampal cortex (PHC), and inferior parietal sulci (IPS0 and IPS1). We averaged the correlation values over the voxels within each ROI resulting in one correlation time series per individual (see Appendix D, spatially restricted searchlight voxel-wise fMRI-MEG fusion method). Figures $2 b$ and $3 b$ compare the ROI-specific time courses for Twin-set 1 and 2. We observe that the two sets result in similar temporal dynamics within the regions of interest across ventral and dorsal pathways. While the analyses reported in Figures $2 b$ and $3 b$ tests for similarity of time courses, we further investigated whether there are any significant dissimilarities. This explicit test however revealed no significant differences (permutation tests, $\mathrm{n}=15$; cluster-definition threshold of $P<0.01$, and cluster size threshold of $P<0.01$ ). This demonstrates the reliability of the fusion method in reproducing similar patterns of neural activity across similar visual experiences.

Next, we performed an ROI-based fMRI-MEG fusion following the method described in [8,9] (see Figure 4a). Studied ROIs (see details in Appendix D) include EVC, ventral regions (VO, TO, PHC) and dorsal regions (IPS0, IPS1, IPS2, and IPS3). As depicted in Figure 4b-e panels, the ROI-based fMRI-MEG fusion results in visually similar time series. Comparison of peak latency times show a peak of response around $120 \mathrm{~ms}$ in EVC and significantly later (two-sided hypothesis test, all $P<0.01$, FDR corrected), around $140 \mathrm{~ms}$ in ventral and dorsal ROIs. The peak latency and onset times with their corresponding 95\% confidence intervals are reported in Table 1.

To assess reproducibility of the full brain $4 \mathrm{D}$ spatiotemporal maps (time $\mathrm{x}$ voxel $\mathrm{x}$ voxel $\mathrm{x}$ voxel) between Twin set 1 and 2, for each participant separately, we compared the voxel-wise fMRI-MEG correlation time series of Twin set 1 with Twin set 2 . This resulted in one 3D reliability (correlation) map per participant (see Appendix E). We assessed significance by permutation tests and corrected for multiple comparisons using the cluster correction method $(\mathrm{n}=15$; cluster-definition threshold of $P<0.01$, and cluster size threshold of $P<0.01$ ). This revealed significant clusters in the reliability map across both the ventral and the dorsal stream (Figure 5). 


\subsection{Generalizability of Similarity-Based fMRI-MEG Fusion Method}

We evaluated the generalizability of the fMRI-MEG similarity-based data fusion method by comparing results across participant groups presented with different images (Twins-Set and ImageNet-Set from Experiment 2 of [9]). We applied the fMRI-MEG similarity-based data fusion method to Twins-set and ImageNet-set, separately. Figures 6 and 7 display the spatiotemporal dynamics of visual perception for the two datasets along the ventral and dorsal visual pathways, respectively, over the first $1000 \mathrm{~ms}$ from stimulus onset. In both cases, the significant signals emerge in EVC around 70-80 ms after stimulus onset and then in the anterior direction along the ventral stream (Figure 6a,c), and across the dorsal stream up to the inferior parietal cortex (Figure $7 \mathrm{a}, \mathrm{c}$ ). We determined significant spatiotemporal correlations with sign-permutation tests $(\mathrm{n}=15 ; \mathrm{P}<0.01$ cluster-definition threshold, $P<0.01$ cluster threshold).

Qualitatively, Figures $6 \mathrm{~b}$ and $7 \mathrm{~b}$ show similar MEG-fMRI time courses for the two datasets (Twins-Set and ImageNet-Set) in EVC, ventral regions (VO and PHC) and dorsal regions (IPS0 and IPS1). Further explicit testing revealed no significant difference between the two sets (permutation tests, $\mathrm{n}=15$; cluster-definition threshold of $P<0.01$, and cluster size threshold of $P<0.01$ ).

To further investigate the similarities and dissimilarities between these two sets of data quantitatively, we performed ROI-based analyses. For each dataset, we correlated ROI-specific fMRI RDMs with time-resolved MEG RDMs resulting in correlation time courses shown in Figure 8a-d. We determined significant time points illustrated with color coded lines below the graphs with sign-permutation tests ( $\mathrm{n}=15 ; P<0.01$ cluster-definition threshold, $P<0.01$ cluster threshold). We observed that in both datasets, correlation time series peak significantly earlier in EVC compared to high level regions in ventral (VO and PHC) and dorsal (IPS0, IPS1, IPS2) pathways (two-sided hypothesis test, all $P<0.01$, FDR corrected) reflecting the established structure of the visual hierarchy. Peak and onset latencies of curves in Figure 8a-d and their corresponding 95\% confidence intervals are reported in Table 2.
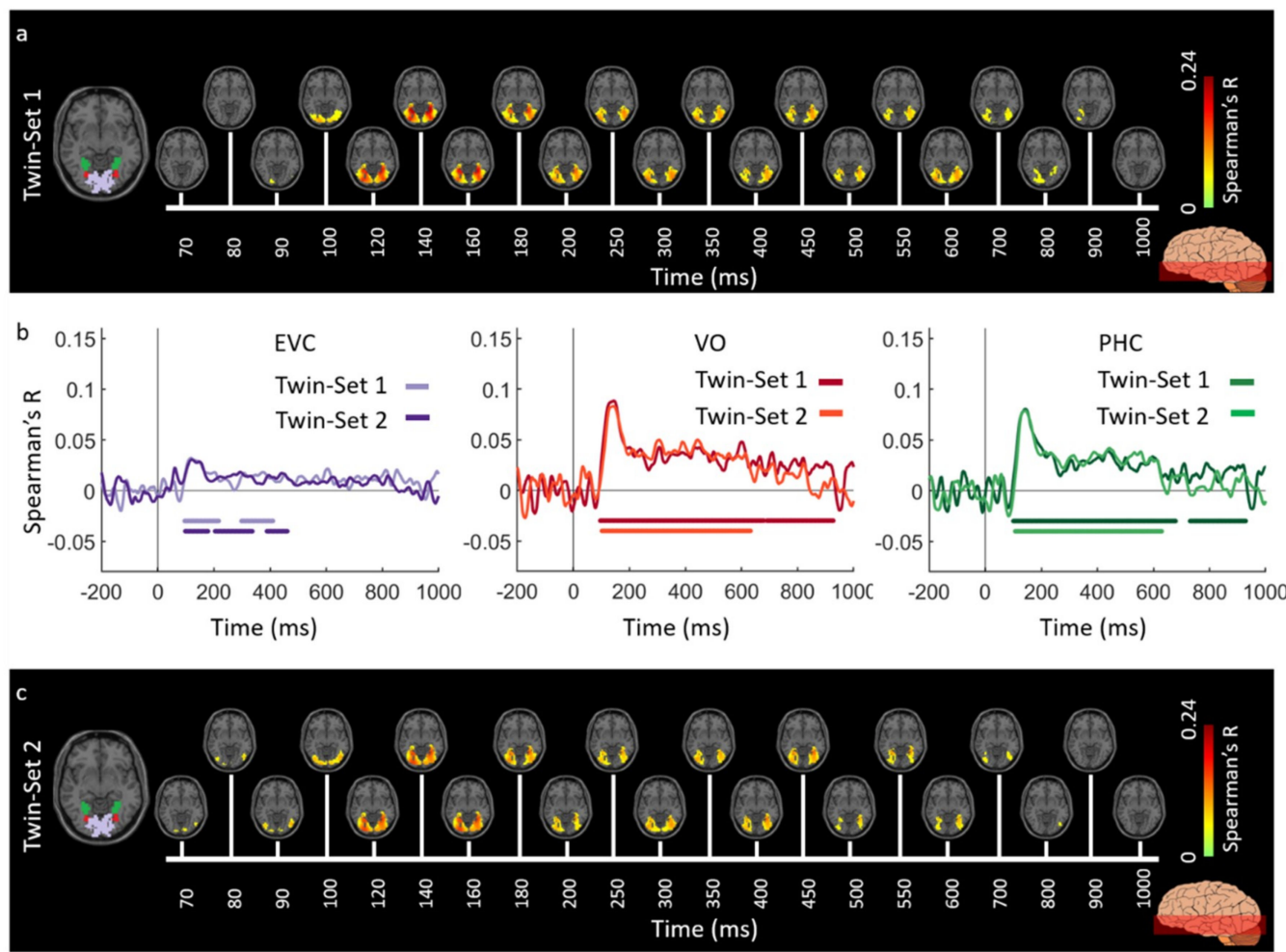

Figure 2. Experiment 1 Twins-set spatiotemporal neural dynamics of vision in ventral stream: (a) An axial slice encompassing early visual cortex (EVC) and ventral ROIs, ventral occipital (VO) area and parahippocampal cortex (PHC). 
The significant correlation maps for Twin-set 1 in this axial slice depicted over time ( $\mathrm{n}=15$, cluster-definition threshold $P<0.001$, cluster threshold $P<0.01$ ); (b) The correlation time series are computed based on spatially restricted searchlight voxel-wise fusion analysis (see Methods). The depicted curves from left to right compare these time series in EVC and ventral ROIs VO and PHC for Twin-set 1 and Twin-set 2. Significant time points depicted with color coded lines below the graphs are determined with sign-permutation tests $(\mathrm{n}=15 ; P<0.01$ cluster-definition threshold, $P<0.01$ cluster threshold); (c) The significant correlation maps for Twin-set 2 in the same axial slice as (a) depicted over time ( $\mathrm{n}=15$, cluster-definition threshold $P<0.001$, cluster threshold $P<0.01$ ).
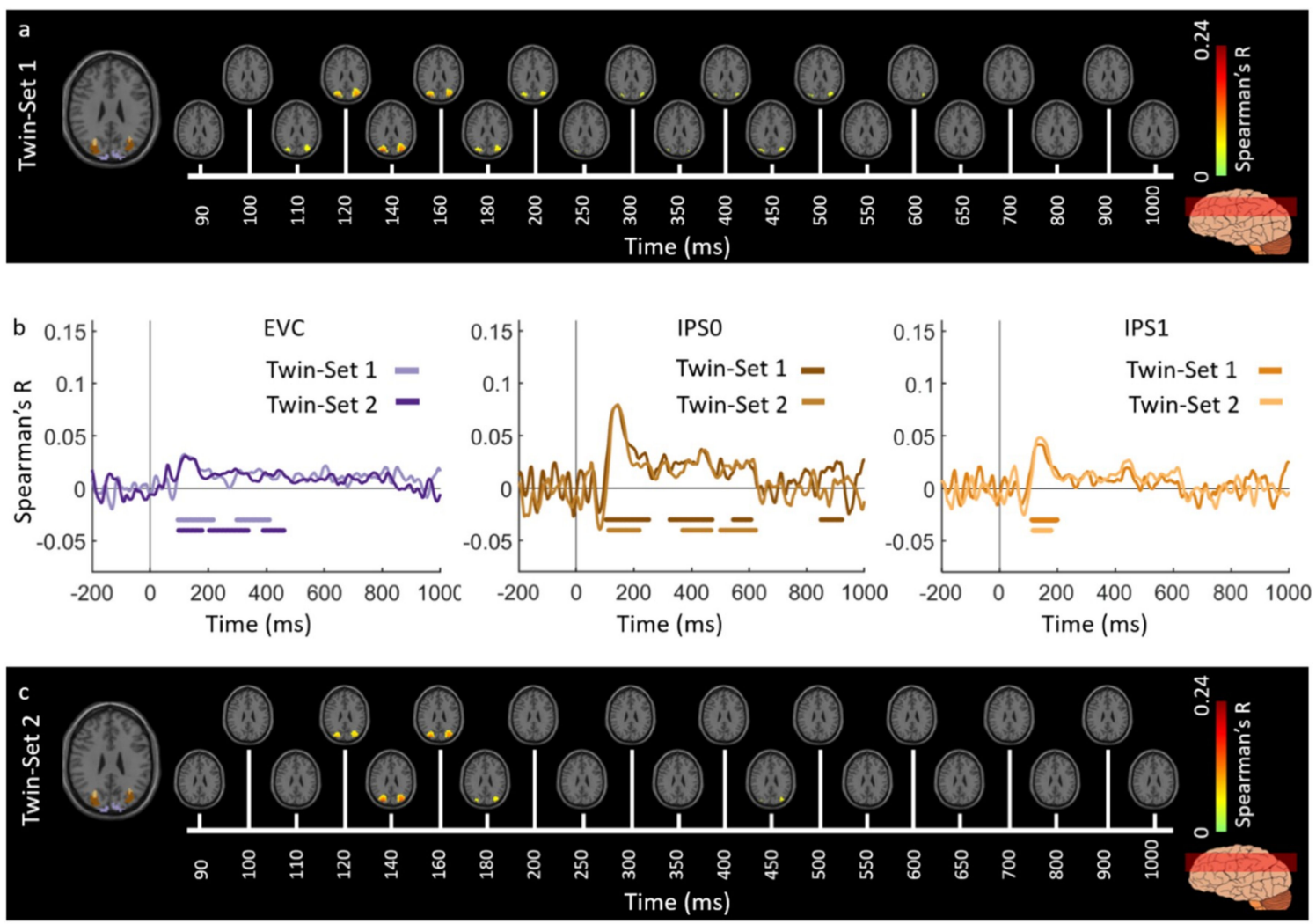

Figure 3. Experiment 1 Twins-set spatiotemporal neural dynamics of vision in dorsal stream: (a) An axial slice encompassing early visual cortex (EVC) and dorsal ROIs, inferior parietal sulcus (IPS0 and IPS1). The significant correlation maps for Twin-set 1 in this axial slice depicted over time (n $=15$, cluster-definition threshold $P<0.001$, cluster threshold $P<0.01$ ); (b) The correlation time series are computed based on spatially restricted searchlight voxel-wise fusion analysis (see Methods). The depicted curves from left to right compare these time series in EVC and dorsal ROIs, IPS0 and IPS1 for Twin-set1 and Twin-set2. Significant time points depicted with color coded lines below the graphs are determined with sign-permutation tests $(n=15 ; P<0.01$ cluster-definition threshold, $P<0.01$ cluster threshold); (c) The significant correlation maps for Twin-set 2 in the same axial slice as (a) depicted over time ( $\mathrm{n}=15$, cluster-definition threshold $P<0.001$, cluster threshold $P<0.01$ ). 

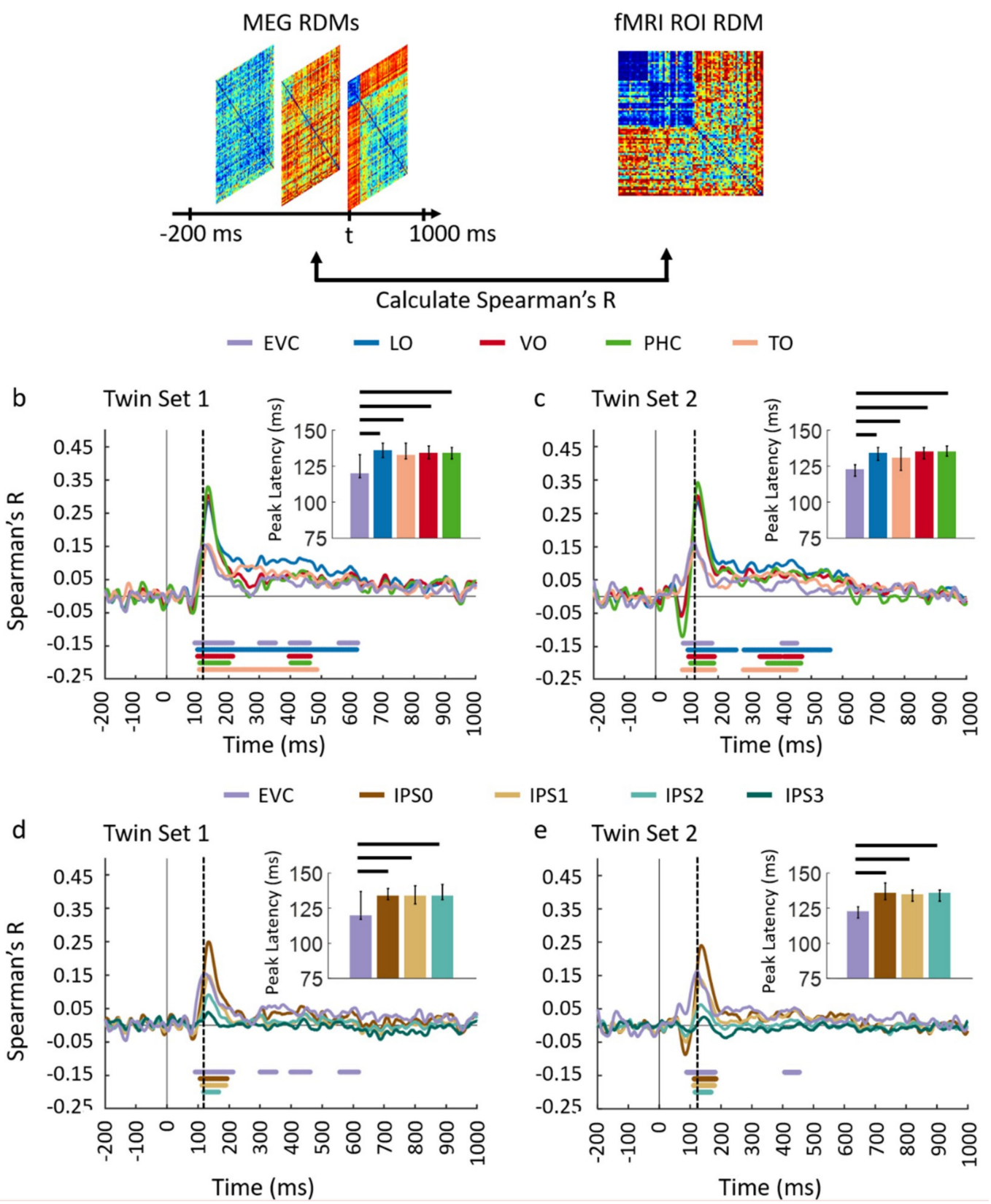

Figure 4. Comparing dorsal and ventral stream neural representations through similarity-based ROI fMRI-MEG fusion. (a) The voxel patterns are extracted from each ROI to construct the fMRI ROI RDM. Then the ROI-specific fMRI RDM was compared with time-resolved MEG RDMs resulting in correlation time series for each region of interest; $(\mathbf{b}, \mathbf{c})$ The $\mathrm{fMRI}-\mathrm{MEG}$ fusion time series are depicted in EVC and ventral ROIs, LO, VO, PHC, and TO for Set 1 and 2, respectively; (d,e) The fMRI-MEG fusion time series are depicted in EVC and dorsal ROIs, IPS0-3 for Twin-set 1 and Twin-set 2, respectively. The color-coded lines below the curves indicate significant time points ( $n=15$, cluster-definition threshold $P<0.01$, cluster threshold $P<0.01$ ) and the dashed vertical line in each plot indicates the peak latency in EVC time series. Peak latency times and their corresponding $95 \%$ confidence intervals for correlation time series in bcde are illustrated with barplots and error bars, respectively. Black lines above the bar indicate significant differences between conditions. 95\% confidence intervals were found with bootstrap tests and barplots were evaluated with two-sided hypothesis tests; false discovery rate corrected at $P<0.05$. 

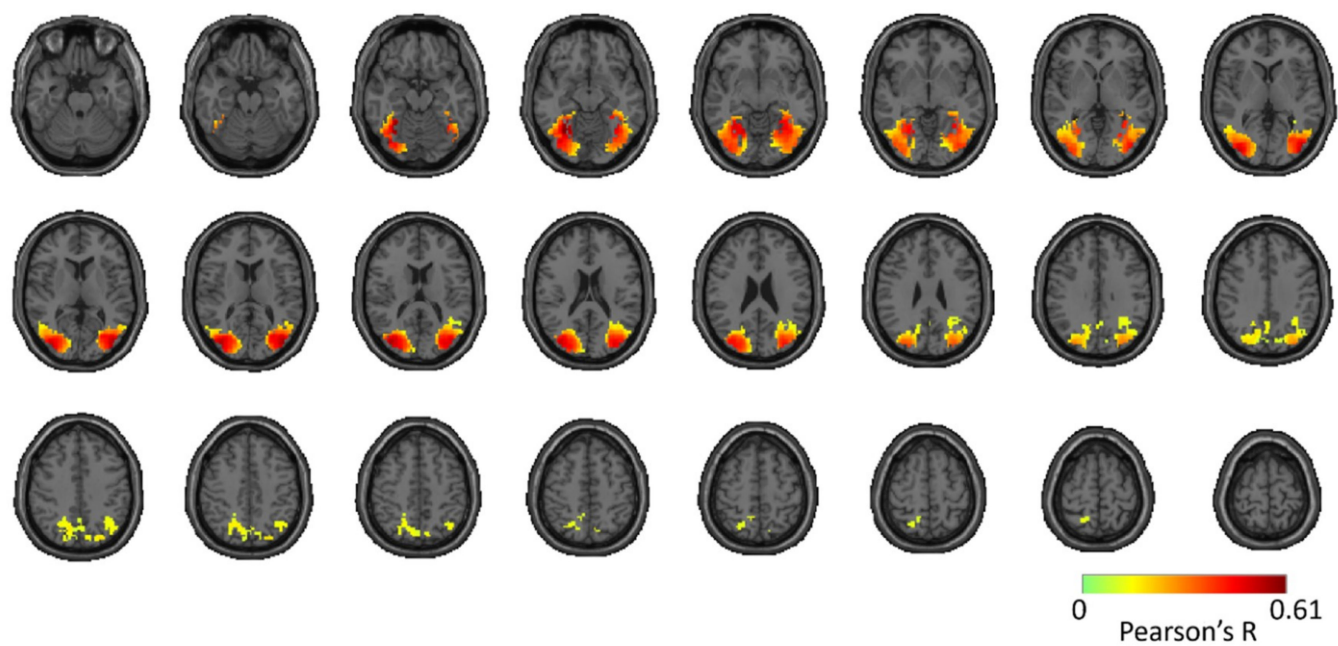

Figure 5. Reliability map of MEG-fMRI fusion time courses for Twin set 1 and Twin set 2 . The figure shows significant clusters projected onto a standard T1 MNI brain. Significant correlations are determined with permutation tests and multiple comparison corrections using cluster correction method ( $\mathrm{n}=15$; cluster-definition threshold of $P<0.01$, and cluster size threshold of $P<0.01$ ).
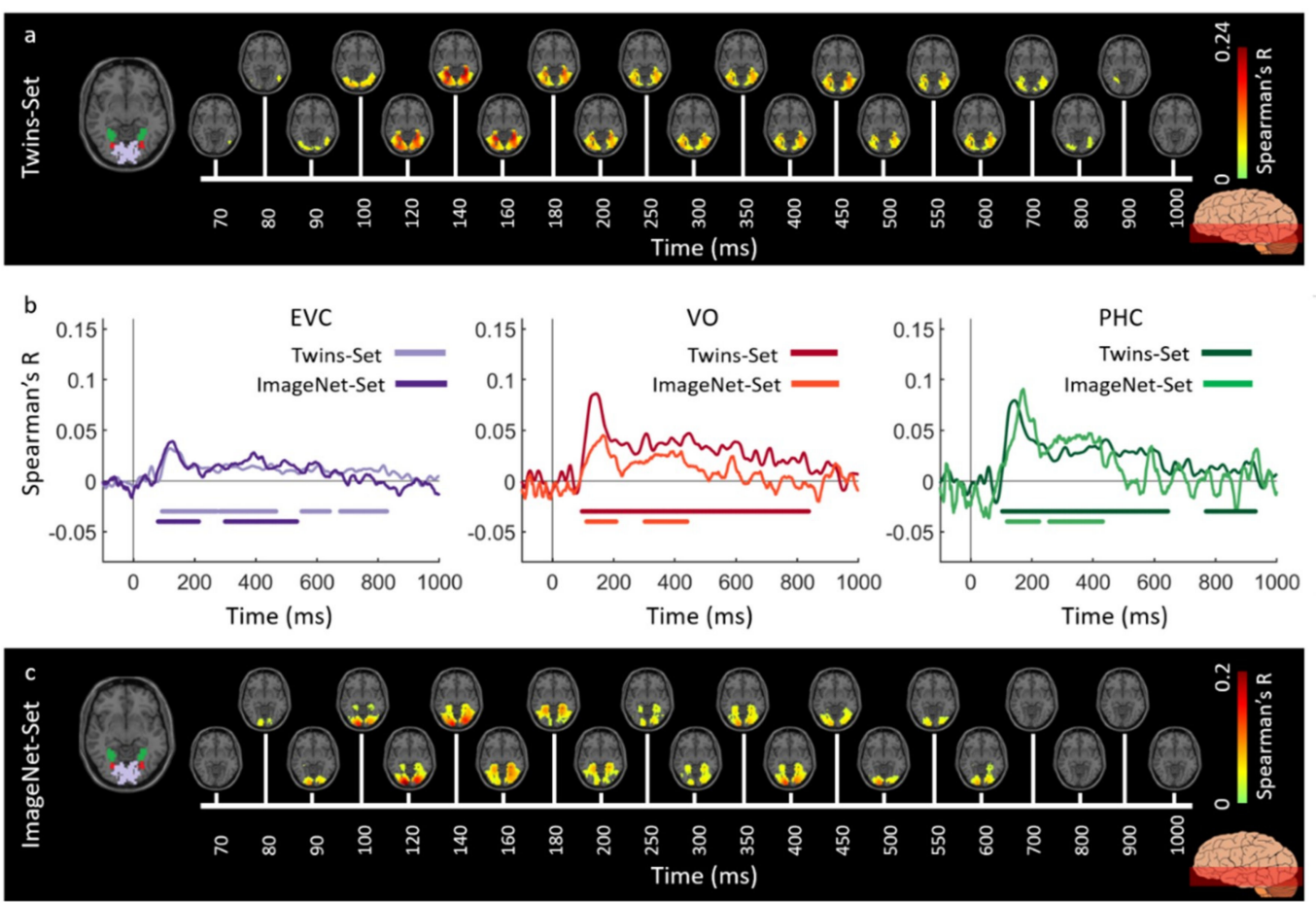

Figure 6. Spatiotemporal neural dynamics of vision in ventral stream: (a) An axial slice encompassing early visual cortex (EVC) and ventral ROIs, occipital (VO) area and parahippocampal cortex (PHC). The significant correlation maps for Twins-set (Experiment 1) in this axial slice depicted over time ( $\mathrm{n}=15$, cluster-definition threshold $P<0.001$, cluster threshold $P<0.01$ ); (b) The correlation time series are computed based on spatially restricted searchlight voxel-wise fusion analysis (see Methods). The depicted curves from left to right compare these time series in EVC and ventral ROIs VO and PHC for Twins-set (Experiment 1) and ImageNet-set (Experiment 2). Significant time points depicted with color coded lines below the graphs are determined with sign-permutation tests $(n=15$; $P<0.01$ cluster-definition threshold, $P<0.01$ cluster threshold); (c) The significant correlation maps for ImageNet-set (Experiment 2 ) in the same axial slice as (a) depicted over time ( $\mathrm{n}=15$, cluster-definition threshold $P<0.001$, cluster threshold $P<0.01$ ). 

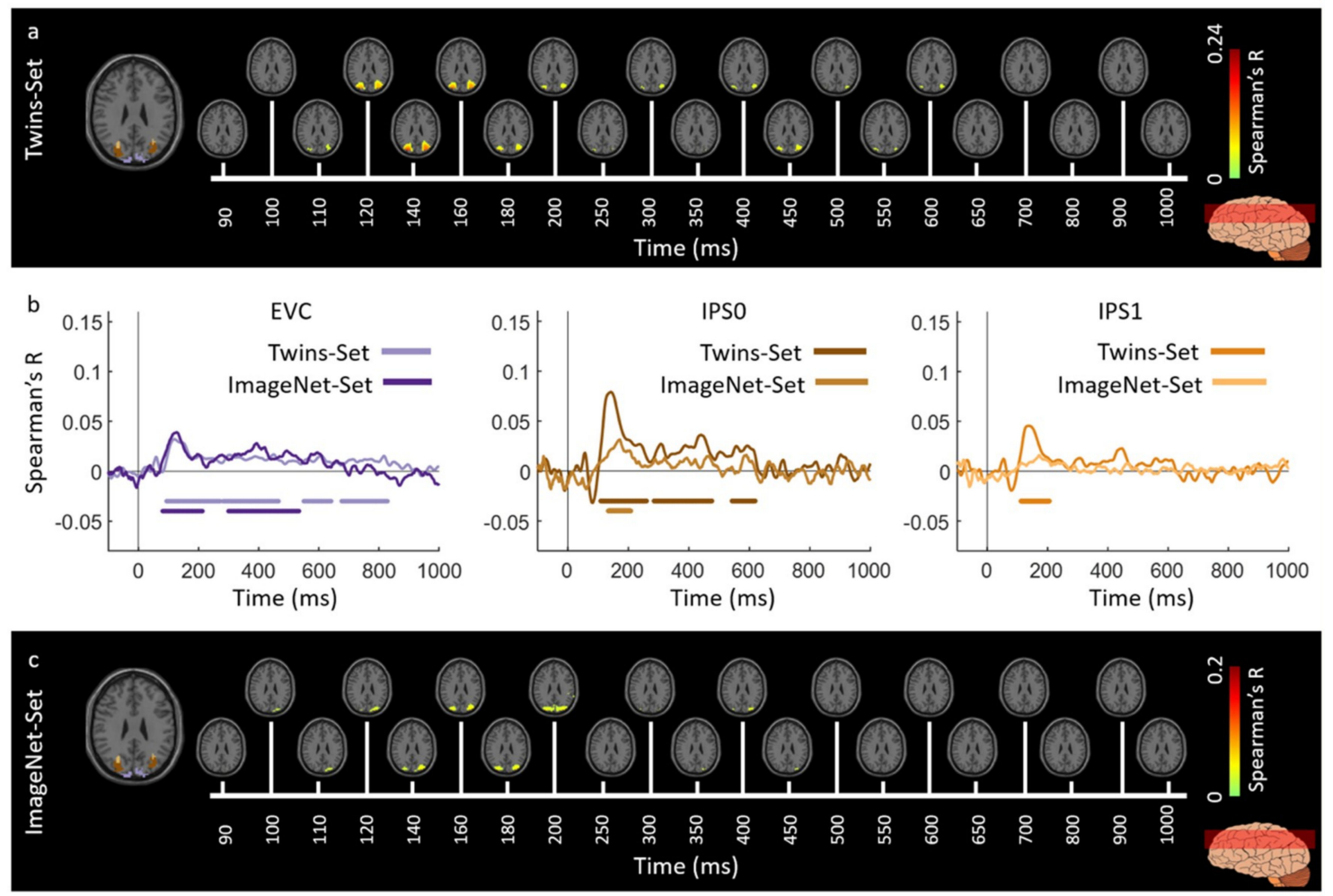

Figure 7. Spatiotemporal neural dynamics of vision in dorsal stream: (a) An axial slice encompassing early visual cortex (EVC) and dorsal ROIs, inferior parietal sulcus (IPS0 and IPS1). The significant correlation maps for Twins-set (Experiment 1) in this axial slice depicted over time ( $\mathrm{n}=15$, cluster-definition threshold $P<0.001$, cluster threshold $P<0.01$ ); (b) The correlation time series are computed based on spatially restricted searchlight voxel-wise fusion analysis (see Methods). The depicted curves from left to right compare these time series in EVC and dorsal ROIs, IPS0 and IPS1 for Twins-set (Experiment 1) and ImageNet-set (Experiment 2). Significant time points depicted with color coded lines below the graphs are determined with sign-permutation tests $(\mathrm{n}=15$; $P<0.01$ cluster-definition threshold, $P<0.01$ cluster threshold); (c) The significant correlation maps for ImageNet-set (Experiment 2) in the same axial slice as (a) depicted over time ( $\mathrm{n}=15$, cluster-definition threshold $P<0.001$, cluster threshold $P<0.01$ ). 

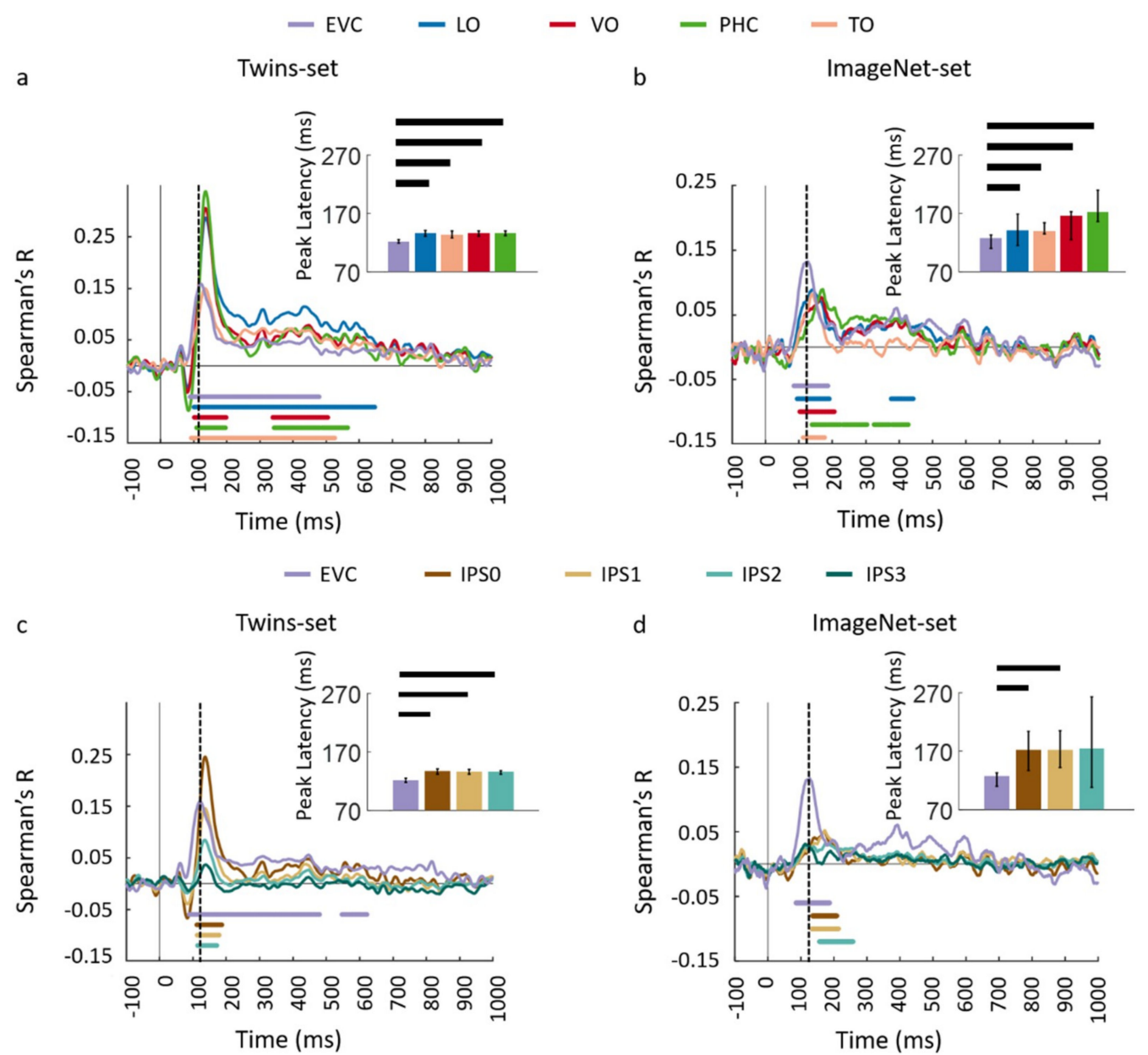

Figure 8. Comparing dorsal and ventral stream neural representations through similarity-based ROI fMRI-MEG fusion. The voxel patterns are extracted from each ROI to construct the fMRI ROI RDM. Then the ROI-specific fMRI RDM was compared with time-resolved MEG RDMs resulting in correlation time series for each region of interest; $(\mathbf{a}, \mathbf{b})$ The fMRI-MEG fusion time series are depicted in EVC and ventral ROIs, LO, VO, PHC, and TO for Twins-set and ImageNet-set, respectively; $(\mathbf{c}, \mathbf{d})$ The fMRI-MEG fusion time series are depicted in EVC and dorsal ROIs, IPS0-3 for Twins-set and ImageNet-set, respectively. The color-coded lines below the curves indicate significant time points ( $\mathrm{n}=15$, cluster-definition threshold $P<0.01$, cluster threshold $P<0.01$ ) and the dashed vertical line in each plot indicates the peak latency in EVC time series. Peak latency times and their corresponding $95 \%$ confidence intervals for correlation time series in bcde are illustrated with barplots and error bars, respectively. Black lines above the bar indicate significant differences between conditions. 95\% confidence intervals were found with bootstrap tests and barplots were evaluated with two-sided hypothesis tests; false discovery rate corrected at $P<0.05$.

Table 1. Peak and onset latency for fMRI-MEG fusion time series in EVC, ventral, and dorsal regions for Twin-Set 1 and Twin-Set 2 (Experiment 1).

\begin{tabular}{ccccc}
\hline \multirow{2}{*}{ Region of Interest } & \multicolumn{2}{c}{ Twin-Set 1 } & \multicolumn{2}{c}{ Twin-Set 2 } \\
\cline { 2 - 5 } & Peak latency (ms) & Onset latency (ms) & Peak latency (ms) & Onset latency (ms) \\
\hline EVC & $120(117-137)$ & $92(85-99)$ & $123(118-126)$ & $90(51-94)$ \\
Dorsal regions & & & & \\
IPS0 & $134(131-139)$ & $107(97-116)$ & $136(131-143)$ & $113(107-118)$ \\
IPS1 & $134(128-141)$ & $113(102-123)$ & $135(130-138)$ & $111(105-116)$ \\
IPS2 & $134(131-142)$ & $118(95-156)$ & $136(130-138)$ & $116(107-126)$ \\
\hline
\end{tabular}


Table 1. Cont.

\begin{tabular}{ccccc}
\hline \multirow{2}{*}{ Region of Interest } & \multicolumn{2}{c}{ Twin-Set 1 } & \multicolumn{2}{c}{ Twin-Set 2 } \\
\cline { 2 - 5 } & Peak latency $(\mathbf{m s})$ & Onset latency $(\mathbf{m s})$ & Peak latency (ms) & Onset latency (ms) \\
\hline Ventral regions & & & & \\
LO & $136(131-141)$ & $101(95-107)$ & $134(129-138)$ & $105(100-108)$ \\
VO & $134(130-139)$ & $100(95-105)$ & $135(130-138)$ & $104(99-111)$ \\
TO & $133(130-141)$ & $104(92-111)$ & $131(122-138)$ & $85(73-101)$ \\
PHC & $134(130-138)$ & $106(99-113)$ & $135(132-139)$ & $112(107-116)$ \\
\hline
\end{tabular}

Values are averaged over $n=15$ subjects with $95 \%$ confidence intervals reported in parentheses.

Table 2. Peak and onset latency for fMRI-MEG fusion time series in EVC, ventral, and dorsal regions for Twins-Set (Experiment 1) and ImageNet-Set (Experiment 2).

\begin{tabular}{ccccc}
\hline \multirow{2}{*}{ Region of Interest } & \multicolumn{2}{c}{ Twins-Set } & \multicolumn{2}{c}{ ImageNet-Set } \\
\cline { 2 - 5 } & Peak latency (ms) & Onset latency (ms) & Peak latency (ms) & Onset latency (ms) \\
\hline EVC & $122(119-125)$ & $89(56-95)$ & $127(110-133)$ & $84(71-91)$ \\
Dorsal regions & & & & $172(137-204)$ \\
IPS0 & $137(131-141)$ & $111(102-117)$ & $133(99-156)$ \\
IPS1 & $136(132-140)$ & $111(103-119)$ & $172(142-205)$ & $137(99-158)$ \\
IPS2 & $136(132-138)$ & $112(100-119)$ & $174(108-263)$ & $103(90-186)$ \\
Ventral regions & & & & \\
LO & $136(131-141)$ & $102(97-107)$ & $141(115-169)$ & $96(87-118)$ \\
VO & $136(131-139)$ & $102(95-109)$ & $166(125-173)$ & $103(95-125)$ \\
TO & $134(128-140)$ & $90(77-100)$ & $139(135-154)$ & $115(95-126)$ \\
PHC & $136(132-139)$ & $109(102-115)$ & $172(156-210)$ & $121(99-159)$ \\
\hline
\end{tabular}

Values are averaged over $n=15$ subjects with $95 \%$ confidence intervals reported in parentheses.

\section{Discussion}

One of the central tenants of science is reproducibility [43-45]. This is especially relevant in cognitive neuroscience, as the surge of new cutting-edge multivariate analysis tools has made it possible to address questions that were previously untestable [41,46]. Here, we have demonstrated the reproducibility of the fMRI-MEG fusion method through the application of two separate experiments, testing the reliability and generalizability of the technique.

The first analysis compared neural data within the same subject groups across Twin image sets, with equalized low-level visual features, and sharing highly similar semantic concepts (e.g., a giraffe for a giraffe, a flower for a flower). We observed that the analysis yielded reliably consistent spatiotemporal dynamics, with each subset showing responses first in the occipital pole, and then signals in the anterior direction, with similar path in both ventral and dorsal streams. The strong agreement between the full brain spatiotemporal maps of the Twin-sets suggests that the signals detected by the fMRI-MEG data fusion method is likely to reliably reflect the representations of the stimulus. This sequence of representational signals followed the established spatial $[47,48]$ and temporal $[19,49]$ patterns associated with hierarchical visual processing.

This high degree of reliability, within subjects, across these separate but matched image-sets has pragmatic consequences in reinforcing the power and confidence of this method. The agreement within results of unimodal neuro-imaging have been shown have low test-retest reliability [29]. This realization has brought about a crisis of confidence in the replicability and reliability of published research findings within neuroscience [50,51]. To mitigate these concerns the generation of new research discoveries requires that findings move beyond drawing conclusions solely on the basis of a single study. As such, we sought to extend this replication by comparing the newly collected Twins-Set data to previously published independent fMRI-MEG fusion data [9]. This analysis focused on the generalizability of this method across subject groups and to a wider range of stimuli. 
Across the two experiments, findings from the full brain correlation maps and spatially restricted ROI analysis revealed that the fMRI-MEG data fusion captures the spatiotemporal dynamic patterns common to visual processing in a manner that generalizes across subjects and natural images. The results of both experiments illustrate the spatiotemporal progression associated with hierarchical visual processing $[6,52,53]$, with significant signals emerging first in early visual areas and then along the ventral and dorsal pathways. Additionally, qualitative comparison of correlation time series in Figures $2 b$ and $3 b$ with Figures $6 b$ and $7 b$ reveals that the response patterns between Twin set 1 and 2 are qualitatively more similar than between the Twins sets and the ImageNet set. This is most likely due to the fact that Twin set 1 and 2 had similar low- and high-level features by design, and both were different from the features of the ImageNet set. Nevertheless, the similarity in spatiotemporal dynamics in the two experiments suggests that the neural dynamics in processing natural images are similar.

We found that overall this generalized response had similar expected spatiotemporal response patterns, but with greater variability in high-level visual areas. Previous work examining the replicability of neuroimaging data has shown that low-level brain functions (motor and sensory tasks), show generally less variance than high level cognitive tasks [54]. Moreover, between subject comparisons show significantly higher variability than within due to the increase sources of noise [55].

Cognitive neuroscientists have many methodological options for studying how experimental variables are systematically related to the spatiotemporal dynamics underlying a cortical process of interest. To date, much of our understanding has been advanced by methods that yield high spatial or temporal resolution, but not both. Separately, the limitations of these modalities in their application to understanding cognition are well known. Here we show that combining these methods through representational similarity analysis provides a reliable and generalizable tool for studying visual perception. This confirmatory replication within and between image-sets and subject-groups demonstrates the replicability of the method in the study of visual processing. By establishing replicability our study increases the trustworthiness of the method, and we hope that this motivates further researchers to use the method in the study of human brain function.

Author Contributions: Conceptualization, Y.M., C.M., R.C., A.O.; investigation, Y.M., C.M., R.C., A.O.; methodology, Y.M., C.M., R.C., A.O.; data analysis, B.L., Y.M.; writing and editing, Y.M., C.M., R.C., A.O.; visualization, Y.M, C.M.; supervision, R.C., A.O.; funding acquisition, R.C., A.O.

Funding: This research was funded by NSF grant number 1532591, in Neural and Cognitive Systems as well as the Vannevar Bush Faculty Fellowship program funded by the ONR grant number N00014-16-1-3116 (to A.O.) and the DFG Emmy Noether Grant CI 241/1-1 (to R.C.).

Acknowledgments: The experiments were conducted at the Athinoula A. Martinos Imaging Center at the McGovern Institute for Brain Research, Massachusetts Institute of Technology. The authors would like to thank Dimitrios Pantazis for helpful discussions. The data will be available online.

Conflicts of Interest: The authors declare no conflict of interest. The funders had no role in the design of the study; in the collection, analyses, or interpretation of data; in the writing of the manuscript, or in the decision to publish the result.

\section{Appendix A}

Twin sets features control: As described in the Methods section the twin sets consists of 78 pairs of natural images. The images were controlled for high-level verbal semantics by matching the verbal semantic description of the main object shown (determined by consensus among authors). Further, images sets were matched for low-level image features (color, luminance, brightness, and spatial frequency) using the natural image statistical toolbox [37-39]. In detail, the toolbox statistically compares several stimulus features such as spatial frequency at different energy levels (spectral energy, Table A1), color (Table A2), brightness and contrast. There were no significant differences between twin set $1 \& 2$ in any feature. 
Table A1. Comparison of the spatial frequency information between the Twin Sets (Experiment 1).

\begin{tabular}{ccccc}
\hline & \multicolumn{4}{c}{ Spectral Energy Level (Spatial Frequency) } \\
\cline { 2 - 5 } & $\mathbf{1 0 \%}$ & $\mathbf{3 0} \%$ & $\mathbf{7 0} \%$ & $\mathbf{9 0} \%$ \\
\hline$t$ value & -0.43 & 0.87 & 0.37 & -0.37 \\
$p$ value & 0.67 & 0.37 & 0.7 & 0.72 \\
\hline
\end{tabular}

Table A2. Comparison of color distribution (RGB and Lab) between the Twin Sets (Experiment 1).

\begin{tabular}{ccccccc}
\hline & \multicolumn{5}{c}{ Color Distribution } \\
\cline { 2 - 6 } & & RGB Space & \multicolumn{4}{c}{ Lab Space (Brightness and Contrast) } \\
\hline \multirow{2}{*}{ value } & 0.65 & G & B & L & a & b \\
$p$ value & 0.51 & 0.99 & 0.71 & 0.87 & -0.80 & 0.05 \\
\hline
\end{tabular}

Experimental Design: In both experiments, images were presented at the center of the screen for $500 \mathrm{~ms}$ with 6 and 4 degrees of visual angle in Experiment 1 and 2, respectively. The task was the same for both experiments: the images were overlaid with a black fixation cross at the center and participants performed an orthogonal vigilance task. The data were collected over one MEG session and two fMRI sessions in both experiments.

MEG session included 25 runs for Experiment 1 and 15 runs for Experiment 2. Images were presented once per run in randomized order with 1-1.2 s stimulus onset asynchrony (SOA) in Experiment 1 and twice per run with 0.9-1 s SOA in Experiment 2. The participants were instructed to press a button and make an eye blink upon detection of a specific image (depicting an eye in Experiment 1 and a paper clip in Experiment 2 presented every 3-5 trials randomly.

The two fMRI sessions consisted of 11-15 runs in Experiment 1 and 9-11 runs in Experiment 2. Images were presented once per run in random order. In both studies 39 null trials were randomly distributed in each run. During the null trials the fixation cross changed color for $100 \mathrm{~ms}$ and subjects were instructed to respond to this color change by pressing a button. The trial duration was $3 \mathrm{~s}$ in both studies. Images were presented for $500 \mathrm{~ms}$ and stimulus onset asynchrony was $3 \mathrm{~s}$, or $6 \mathrm{~s}$ (if a preceding null trial).

\section{Appendix B}

\section{MEG Acquisition and Analysis}

MEG data were acquired from a 306-channel Elekta neuromag TRIUX system with sampling rate of $1 \mathrm{kHz}$ and filtered by a 0.03 to $330 \mathrm{~Hz}$ band-pass filter. Participants' head position was measured prior and during the recording with 5 coils attached to their head. A maxfilter was applied for temporal source space separation and correcting for head movements [56,57]. Data were analyzed using Brainstorm software [58] to extract trials from $-200 \mathrm{~ms}$ to $1000 \mathrm{~ms}$ with respect to image onset in Experiment 1 and from $-100 \mathrm{~ms}$ to $1000 \mathrm{~ms}$ relative to image onset in Experiment 2. The mean of the baseline was removed and the data were smoothed with a $30 \mathrm{~Hz}$ low-pass filter. For each condition and participant, we obtained 25 trials in Experiment 1 and 30 trials in Experiment 2.

Multivariate pattern analysis was used to determine the similarity relations between visual conditions (Figure 1b). At each time point $t$, MEG sensor data were arranged in pattern vectors resulting in $\mathrm{N}$ vectors per condition at that time point with $\mathrm{N}$ being the number of trials per condition. Then, for each pair of conditions a support vector machine (SVM) classifier was trained to discriminate the conditions based on the MEG pattern vectors at each time point. The performance of the classifier computed with leave-one-out cross validation procedure was considered as a measure of dissimilarity between those pairs of conditions and used to construct a $C \times C(C=156$ in Experiment 1 and $C=118$ 
in Experiment 2) representational dissimilarity matrix (RDM) at each time point. Each row and column in this matrix are indexed by a specific condition (image) and each matrix entry shows how dissimilar the corresponding conditions are based on the MEG signals at a specific time point. These matrices are symmetric with an undefined diagonal. To overcome computational complexity and reduce noise, we subaveraged trials for each experimental condition (image separately) with random assignment of trials to bins of 3 in Experiment 1, and bins of 5 in Experiment 2, before entering them into multivariate analysis.

\section{fMRI Acquisition and Analysis}

The MRI data of both experiments were acquired with a 3T Trio Siemens scanner using a 32-channel head coil. The structural images were acquired using a standard T1-weighted sequence (192 sagittal slices, FOV $=256 \mathrm{~mm}, \mathrm{TR}=1900 \mathrm{~ms}, \mathrm{TE}=2.52 \mathrm{~ms}$, flip angle $=9^{\circ}$ ).

Functional data of Experiment 1 was acquired over 11-15 runs of 305 volumes in two sessions with gradient-echo EPI sequence: $\mathrm{TR}=2000 \mathrm{~ms}, \mathrm{TE}=29 \mathrm{~ms}$, flip angle $=90^{\circ}, \mathrm{FOV}$ read $=200 \mathrm{~mm}$, FOV phase $=100 \%$, bandwidth $2368 \mathrm{~Hz} / \mathrm{Px}$, resolution $=3 \times 3 \times 3 \mathrm{~mm}$, slice gap 20\%, slices $=33$, ascending interleaved acquisition). Functional data of Experiment 2 was acquired over 9-11 runs of 648 volumes in two sessions with gradient-echo EPI sequence: $\mathrm{TR}=750 \mathrm{~ms}, \mathrm{TE}=30 \mathrm{~ms}$, flip angle $=61^{\circ}$, FOV read $=192 \mathrm{~mm}$, FOV phase $=100 \%$ with a partial fraction of $6 / 8$, through-plane acceleration factor 3 , bandwidth $1816 \mathrm{~Hz} / \mathrm{Px}$, resolution $=3 \times 3 \times 3 \mathrm{~mm}$, slice gap $20 \%$, slices $=33$, ascending interleaved acquisition).

The fMRI data in both studies were preprocessed using SPM software. For each participant, fMRI data were slice-time corrected, realigned and co-registered to the T1 structural scan of the first session, and finally normalized to the standard MNI space. We applied a general linear model (GLM) to estimate the fMRI responses to the 156 and 118 image conditions in Experiment 1 and 2, respectively. Stimulus onsets and durations, as well as motion and run regressors were included in the GLM. All the regressors were convolved with a hemodynamic response function (canonical HRF) with time resolution of $T R / N$ and onset of the first slice ( $T R=2000 \mathrm{~ms}, \mathrm{~N}=33$ slices). By contrasting each condition against the implicit baseline, we converted the condition-specific GLM estimates into $t$-value maps (resulting in 156 condition-specific t-maps for Experiment 1 and 118 condition-specific t-maps for Experiment 2).

We then applied the searchlight analysis method to create the fMRI RDMs $[59,60]$ for each subject, separately. At each voxel $v$, condition-specific t-value pattern vectors were extracted in a sphere centered at voxel $v$ with a 4 -voxel radius. Dissimilarities between these pattern vectors were computed in a pairwise manner (1-Pearson's R). Then these dissimilarities were entered into an RDM matrix in which its rows and columns were indexed by the image conditions. This process resulted in a $156 \times 156 \mathrm{RDM}$ at each voxel for fMRI data of each subject in Experiment 1 and a $118 \times 118 \mathrm{RDM}$ at each voxel for fMRI data of each subject in Experiment 2.

\section{Appendix C}

Full Brain fMRI-MEG Fusion: The assumption in the fusion analysis is that the pairwise condition-specific relations (in the form of RDM representations) are preserved across MEG and fMRI data patterns. For example, if two images result in similar patterns in MEG data, they produce similar patterns in fMRI data as well. Comparison of pairwise similarity relations across MEG and fMRI makes it possible to relate representations at specific time points (in MEG) with specific locations (in fMRI). For both datasets, we performed within subject analysis comparing averaged MEG RDMs over the 15 subjects, with subject-specific fMRI RDMs. We computed the correlations (Spearman's R) between MEG RDMs at each time point and fMRI RDMs at each voxel. These computations resulted in a 3D correlation map over the whole brain at each time point. Repeating the analysis over time yielded a spatiotemporally resolved view of visual information in the brain. We used Spearman rather than 
Pearson correlation to compare MEG-fMRI responses because we assume that the values compared are monotonically, rather than linearly related to each other.

\section{Appendix D}

Region-of-Interest Analysis

Spatially Restricted Searchlight Voxel-wise fMRI-MEG Fusion: This analysis followed the regular voxel-wise full brain fMRI-MEG fusion but in spatially restricted regions of the brain. In detail, the search-light method was performed within the ROI and an RDM matrix was created at each specific voxel by comparing condition-specific fMRI pattern responses within the searchlight sphere centered at the voxel (1-Pearson's R). Then the time-resolved MEG RDMs were compared with these fMRI RDMs (Spearman's R) resulting in correlation time series within the ROI which were then averaged resulting in one correlation time-course per ROI and per subject. This ROI analysis method picks out the spatiotemporal dynamics in particular cortical regions as resolved with the searchlight fMRI-MEG fusion method. This method is directly comparable with the full brain fusion analysis and it is a convenient way to quantitatively compare the spatiotemporal dynamics of fusion movies within specific brain regions.

ROI-based fMRI-MEG Fusion: This analysis is the previously established way of performing ROI based fusion and is directly comparable with the original fusion studies $[8,9]$. We extracted the voxel patterns within each ROI and then computed the condition-specific pairwise dissimilarities (1-Pearson's R) to create a single RDM matrix per ROI. We obtained one fMRI RDM for each ROI per subject. The fMRI ROI RDMs were averaged over subjects and then compared with subject-specific MEG RDMs over time by computing their Spearman's R correlations. This process results in time-course of MEG-fMRI fusion in each ROI per subject.

Specifically, we extracted the following ROIs: one early visual cortex (combining V1, V2, V3), four ventral visual areas (lateral occipital (LO1\&2), temporal occipital (TO), ventral occipital (VO1\&2), and parahippocampal cortex (PHC1\&2) and four parietal areas (intraparietal cortex including IPS0, IPS1, IPS2, and IPS3). The ROI definition followed [9] based on the probabilistic maps of [61].

\section{Appendix E}

\section{Reliability Maps}

To assess the reproducibility of the full brain fMRI-MEG fusion method, we computed participant-specific reliability maps. For this, at each voxel we compared the MEG-fMRI correlation time series of Twin set 1 with the corresponding time series of Twin set 2 by computing Pearson's correlations. This yielded a 3D reliability (correlation) map per individual. We determined significance with permutation tests and corrected for multiple comparisons using cluster correction.

\section{Appendix F}

\section{Statistical Testing}

We used non-parametric permutation-based cluster-size inference $[62,63]$ as used also previously in the original fusion studies [8,9]. In detail, we first averaged the $4 \mathrm{D}$ subject-specific spatiotemporal maps over subjects resulting in a mean $4 \mathrm{D}$ correlation map. We then constructed an empirical distribution of the null hypothesis using the voxel values of the mean correlation map in the baseline period, i.e., from -200 to $0 \mathrm{~ms}$ for Twin Set 1 and 2, and from -100 to $0 \mathrm{~ms}$ for ImageNet set. The right-sided $99.9 \%$ threshold (i.e., $P=0.001$ ) was determined as the cluster-definition threshold. To estimate a permutation-based maximal cluster size threshold, the sign of subject-specific data points was shuffled randomly (1000 iterations), the data was averaged over subjects, and 4D clusters were 
determined by spatial and temporal contiguity at the cluster-definition threshold. We then constructed an empirical distribution of maximal cluster size over permutation sampling and determined the $99 \%$ threshold (i.e., cluster size threshold at $P=0.01$ ). We reported clusters as significant if they exceed this threshold.

For peak and onset latency of the time series statistical assessments, bootstrap tests were performed. We bootstrapped the subject-specific time series for 1000 times to estimate an empirical distribution over peak and onset latency of the subject-averaged time courses and use it to define $95 \%$ confidence intervals. For peak to peak latency comparisons, 1000 bootstrapped samples of two peak differences were obtained. The null hypothesis was rejected if the $95 \%$ confidence interval of the peak latency differences did not include zero.

\section{References}

1. Malach, R.; Reppas, J.B.; Benson, R.R.; Kwong, K.K.; Jiang, H.; Kennedy, W.A.; Ledden, P.J; Brady, T.J.; Rosen, B.R.; Tootell, R.B. Object-related activity revealed by functional magnetic resonance imaging in human occipital cortex. Proc. Natl. Acad. Sci. USA 1995, 92, 8135-8139. [CrossRef] [PubMed]

2. Grill-Spector, K.; Kourtzi, Z.; Kanwisher, N. The lateral occipital complex and its role in object recognition. Vis. Res. 2001, 41, 1409-1422. [CrossRef]

3. Johnson, J.S.; Olshausen, B.A. Timecourse of neural signatures of object recognition. J. Vis. 2003, 3, 4 . [CrossRef]

4. Op de Beeck, H.P.; Baker, C.I. The neural basis of visual object learning. Trends Cogn. Sci. 2010, 14, 22-30. [CrossRef] [PubMed]

5. Mullin, C.R.; Steeves, J.K.E. Consecutive TMS-fMRI Reveals an Inverse Relationship in BOLD Signal between Object and Scene Processing. J. Neurosci. 2013, 33, 19243-19249. [CrossRef] [PubMed]

6. DiCarlo, J.J.; Zoccolan, D.; Rust, N.C. How Does the Brain Solve Visual Object Recognition? Neuron 2012, 73, 415-434. [CrossRef] [PubMed]

7. Konkle, T.; Oliva, A. A Real-World Size Organization of Object Responses in Occipitotemporal Cortex. Neuron 2012, 74, 1114-1124. [CrossRef]

8. Cichy, R.M.; Pantazis, D.; Oliva, A. Resolving human object recognition in space and time. Nat. Neurosci. 2014, 17, 455-462. [CrossRef]

9. Cichy, R.M.; Pantazis, D.; Oliva, A. Similarity-Based Fusion of MEG and fMRI Reveals Spatio-Temporal Dynamics in Human Cortex During Visual Object Recognition. Cereb. Cortex 2016, 26, 3563-3579. [CrossRef]

10. Dale, A.M.; Halgren, E. Spatiotemporal mapping of brain activity by integration of multiple imaging modalities. Curr. Opin. Neurobiol. 2001, 11, 202-208. [CrossRef]

11. Debener, S.; Ullsperger, M.; Siegel, M.; Engel, A.K. Single-trial EEG-fMRI reveals the dynamics of cognitive function. Trends Cogn. Sci. 2006, 10, 558-563. [CrossRef] [PubMed]

12. Rosa, M.J.; Daunizeau, J.; Friston, K.J. EEG-fMRI Integration: A Critical Review of Biophysical Modeling and Data Analysis Approaches. J. Integr. Neurosci. 2010, 09, 453-476. [CrossRef]

13. Huster, R.J.; Debener, S.; Eichele, T.; Herrmann, C.S. Methods for Simultaneous EEG-fMRI: An Introductory Review. J. Neurosci. 2012, 32, 6053-6060. [CrossRef] [PubMed]

14. Jorge, J.; van der Zwaag, W.; Figueiredo, P. EEG-fMRI integration for the study of human brain function. NeuroImage 2014, 102, 24-34. [CrossRef] [PubMed]

15. Sui, J.; Adali, T.; Yu, Q.; Chen, J.; Calhoun, V.D. A review of multivariate methods for multimodal fusion of brain imaging data. J. Neurosci. Methods 2012, 204, 68-81. [CrossRef] [PubMed]

16. Khaligh-Razavi, S.-M.; Cichy, R.M.; Pantazis, D.; Oliva, A. Tracking the Spatiotemporal Neural Dynamics of Real-world Object Size and Animacy in the Human Brain. J. Cogn. Neurosci. 2018, 30, 1559-1576. [CrossRef] [PubMed]

17. Mohsenzadeh, Y.; Qin, S.; Cichy, R.M.; Pantazis, D. Ultra-Rapid serial visual presentation reveals dynamics of feedforward and feedback processes in the ventral visual pathway. eLife 2018, 7, 1-23. [CrossRef] [PubMed]

18. Hebart, M.N.; Bankson, B.B.; Harel, A.; Baker, C.I.; Cichy, R.M. The representational dynamics of task and object processing in humans. eLife 2018, 7, 1-22. [CrossRef]

19. Thorpe, S.; Fize, D.; Marlot, C. Speed of processing in the human visual system. Nature 1996, 381, 520-522. [CrossRef] 
20. VanRullen, R.; Thorpe, S.J. The Time Course of Visual Processing: From Early Perception to Decision-Making. J. Cogn. Neurosci. 2001, 13, 454-461. [CrossRef]

21. Mormann, F.; Kornblith, S.; Quiroga, R.Q.; Kraskov, A.; Cerf, M.; Fried, I.; Koch, C. Latency and Selectivity of Single Neurons Indicate Hierarchical Processing in the Human Medial Temporal Lobe. J. Neurosci. 2008, 28, 8865-8872. [CrossRef] [PubMed]

22. Liu, H.; Agam, Y.; Madsen, J.R.; Kreiman, G. Timing, Timing, Timing: Fast Decoding of Object Information from Intracranial Field Potentials in Human Visual Cortex. Neuron 2009, 62, 281-290. [CrossRef] [PubMed]

23. Isik, L.; Meyers, E.M.; Leibo, J.Z.; Poggio, T. The dynamics of invariant object recognition in the human visual system. J. Neurophysiol. 2014, 111, 91-102. [CrossRef] [PubMed]

24. Carlson, T.; Tovar, D.A.; Alink, A.; Kriegeskorte, N. Representational dynamics of object vision: The first 1000 ms. J. Vis. 2013, 13, 1. [CrossRef] [PubMed]

25. Contini, E.W.; Wardle, S.G.; Carlson, T.A. Decoding the time-course of object recognition in the human brain: From visual features to categorical decisions. Neuropsychologia 2017, 105, 165-176. [CrossRef] [PubMed]

26. Lowe, M.X.; Rajsic, J.; Ferber, S.; Walther, D.B. Discriminating scene categories from brain activity within 100 milliseconds. Cortex 2018, 106, 275-287. [CrossRef]

27. Simmons, J.P.; Nelson, L.D.; Simonsohn, U. False-Positive Psychology: Undisclosed Flexibility in Data Collection and Analysis Allows Presenting Anything as Significant. Psychol. Sci. 2011, 22, 1359-1366. [CrossRef]

28. Pashler, H.; Harris, C.R. Is the Replicability Crisis Overblown? Three Arguments Examined. Perspect. Psychol. Sci. 2012, 7, 531-536. [CrossRef]

29. Bennett, C.M.; Miller, M.B. fMRI reliability: Influences of task and experimental design. Cogn. Affect. Behav. Neurosci. 2013, 13, 690-702. [CrossRef]

30. Kanwisher, N.; McDermott, J.; Chun, M.M. The Fusiform Face Area: A Module in Human Extrastriate Cortex Specialized for Face Perception. J. Neurosci. 1997, 17, 4302-4311. [CrossRef]

31. Epstein, R.; Harris, A.; Stanley, D.; Kanwisher, N. The Parahippocampal Place Area: Recognition, Navigation, or Encoding? Neuron 1999, 23, 115-125. [CrossRef]

32. Epstein, R.; Kanwisher, N. A cortical representation of the local visual environment. Nature 1998, 392, 598-601. [CrossRef] [PubMed]

33. Downing, P.E. A Cortical Area Selective for Visual Processing of the Human Body. Science 2001, 293, 2470-2473. [CrossRef] [PubMed]

34. Grill-Spector, K.; Kushnir, T.; Edelman, S.; Avidan, G.; Itzchak, Y.; Malach, R. Differential Processing of Objects under Various Viewing Conditions in the Human Lateral Occipital Complex. Neuron 1999, 24, 187-203. [CrossRef]

35. Dilks, D.D.; Julian, J.B.; Paunov, A.M.; Kanwisher, N. The Occipital Place Area Is Causally and Selectively Involved in Scene Perception. J. Neurosci. 2013, 33, 1331-1336. [CrossRef] [PubMed]

36. Khosla, A.; Raju, A.S.; Torralba, A.; Oliva, A. Understanding and Predicting Image Memorability at a Large Scale. In Proceedings of the 2015 IEEE International Conference on Computer Vision (ICCV), Santiago, Chile, 7-13 December 2015; pp. 2390-2398.

37. Torralba, A.; Oliva, A. Statistics of natural image categories. Netw. Comput. Neural Syst. 2003, 14, $391-412$. [CrossRef]

38. Bainbridge, W.A.; Oliva, A. A toolbox and sample object perception data for equalization of natural images. Data Brief 2015, 5, 846-851. [CrossRef] [PubMed]

39. Park, S.; Konkle, T.; Oliva, A. Parametric Coding of the Size and Clutter of Natural Scenes in the Human Brain. Cereb. Cortex 2015, 25, 1792-1805. [CrossRef] [PubMed]

40. Deng, J.; Dong, W.; Socher, R.; Li, L.-J.; Li, K.; Fei-Fei, L. ImageNet: A Large-Scale Hierarchical Image Database. In Proceedings of the 2009 IEEE Conference on Computer Vision and Pattern Recognition, Miami, FL, USA, 20-25 June 2009.

41. Kriegeskorte, N. Representational similarity analysis-Connecting the branches of systems neuroscience. Front. Syst. Neurosci. 2008, 2, 4. [CrossRef]

42. Kriegeskorte, N.; Kievit, R.A. Representational geometry: Integrating cognition, computation, and the brain. Trends Cogn. Sci. 2013, 17, 401-412. [CrossRef]

43. John, L.K.; Loewenstein, G.; Prelec, D. Measuring the Prevalence of Questionable Research Practices With Incentives for Truth Telling. Psychol. Sci. 2012, 23, 524-532. [CrossRef] [PubMed] 
44. Maxwell, S.E.; Lau, M.Y.; Howard, G.S. Is psychology suffering from a replication crisis? What does "failure to replicate" really mean? Am. Psychol. 2015, 70, 487-498. [CrossRef] [PubMed]

45. Munafò, M.R.; Nosek, B.A.; Bishop, D.V.M.; Button, K.S.; Chambers, C.D.; Percie du Sert, N.; Simonsohn, U.; Wagenmakers, E.-J.; Ware, J.J.; Ioannidis, J.P.A. A manifesto for reproducible science. Nat. Hum. Behav. 2017, 1, 0021. [CrossRef]

46. Haynes, J.-D.; Rees, G. Decoding mental states from brain activity in humans. Nat. Rev. Neurosci. 2006, 7, 523-534. [CrossRef]

47. Grill-Spector, K.; Malach, R. THE HUMAN VISUAL CORTEX. Annu. Rev. Neurosci. 2004, 27, $649-677$. [CrossRef] [PubMed]

48. Op de Beeck, H.P.; Haushofer, J.; Kanwisher, N.G. Interpreting fMRI data: Maps, modules and dimensions. Nat. Rev. Neurosci. 2008, 9, 123-135. [CrossRef] [PubMed]

49. Amano, K. Estimation of the Timing of Human Visual Perception from Magnetoencephalography. J. Neurosci. 2006, 26, 3981-3991. [CrossRef] [PubMed]

50. Ioannidis, J.P.A. Why Most Published Research Findings Are False. PLoS Med. 2005, 2, 6. [CrossRef] [PubMed]

51. Button, K.S.; Ioannidis, J.P.A.; Mokrysz, C.; Nosek, B.A.; Flint, J.; Robinson, E.S.J.; Munafò, M.R. Power failure: Why small sample size undermines the reliability of neuroscience. Nat. Rev. Neurosci. 2013, 14, 365-376. [CrossRef] [PubMed]

52. Riesenhuber, M.; Poggio, T. Neural mechanisms of object recognition. Curr. Opin. Neurobiol. 2002, 12, 162-168. [CrossRef]

53. Serre, T.; Oliva, A.; Poggio, T. A feedforward architecture accounts for rapid categorization. Proc. Natl. Acad. Sci. USA 2007, 104, 6424-6429. [CrossRef] [PubMed]

54. Caceres, A.; Hall, D.L.; Zelaya, F.O.; Williams, S.C.R.; Mehta, M.A. Measuring fMRI reliability with the intra-class correlation coefficient. NeuroImage 2009, 45, 758-768. [CrossRef] [PubMed]

55. Miller, M.B.; Van Horn, J.D. Individual variability in brain activations associated with episodic retrieval: A role for large-scale databases. Int. J. Psychophysiol. 2007, 63, 205-213. [CrossRef] [PubMed]

56. Taulu, S.; Kajola, M.; Simola, J. Suppression of Interference and Artifacts by the Signal Space Separation Method. Brain Topogr. 2003, 16, 269-275. [CrossRef]

57. Taulu, S.; Simola, J. Spatiotemporal signal space separation method for rejecting nearby interference in MEG measurements. Phys. Med. Biol. 2006, 51, 1759-1768. [CrossRef] [PubMed]

58. Tadel, F.; Baillet, S.; Mosher, J.C.; Pantazis, D.; Leahy, R.M. Brainstorm: A User-Friendly Application for MEG/EEG Analysis. Comput. Intell. Neurosci. 2011, 2011, 1-13. [CrossRef] [PubMed]

59. Kriegeskorte, N.; Goebel, R.; Bandettini, P. Information-based functional brain mapping. Proc. Natl. Acad. Sci. USA 2006, 103, 3863-3868. [CrossRef]

60. Haynes, J.-D.; Sakai, K.; Rees, G.; Gilbert, S.; Frith, C.; Passingham, R.E. Reading Hidden Intentions in the Human Brain. Curr. Biol. 2007, 17, 323-328. [CrossRef]

61. Wang, L.; Mruczek, R.E.B.; Arcaro, M.J.; Kastner, S. Probabilistic Maps of Visual Topography in Human Cortex. Cereb. Cortex 2015, 25, 3911-3931. [CrossRef]

62. Maris, E.; Oostenveld, R. Nonparametric statistical testing of EEG- and MEG-data. J. Neurosci. Methods 2007, 164, 177-190. [CrossRef]

63. Pantazis, D.; Nichols, T.E.; Baillet, S.; Leahy, R.M. A comparison of random field theory and permutation methods for the statistical analysis of MEG data. NeuroImage 2005, 25, 383-394. [CrossRef] [PubMed]

(C) 2019 by the authors. Licensee MDPI, Basel, Switzerland. This article is an open access article distributed under the terms and conditions of the Creative Commons Attribution (CC BY) license (http://creativecommons.org/licenses/by/4.0/). 